\title{
Efecto de dosis de potasio en el cultivo de ají paprika (Capsicum annuum I.) variedad papri king
}

\section{Effect of potassium dose in the cultivation of paprika pepper (Capsicum annuum l.) variety papri king}

Juan Jorge Maguiña Aguirre ${ }^{1}$, Dante Daniel Cruz Nieto², José Antonio Legua Cárdenas², Ronald Fernando Rodríguez Espinoza ${ }^{3}$, Yasmin Jesús Vélez Chang ${ }^{2}$, Luis Alberto Torres Ruedas ${ }^{2}$

\section{RESUMEN}

El objetivo fue determinar que dosis de potasio es adecuada para obtener un mayor rendimiento de ají paprika en el distrito y provincia de Barranca. Se instalaron cuatro bloques y cuatro tratamientos: $\mathrm{T} 1=200, T 2=150, T 3=100$ y T4 $=0 \mathrm{~kg} / \mathrm{ha}$. Se empleó el Diseño de Bloques Completamente al Azar y se realizó el análisis de varianza al $5 \%$. El tratamiento T1 sobresalió en las características físicas del ají paprika: altura de la planta ( $77,95 \mathrm{~cm})$, número de frutos por planta (15 unidades), peso del fruto seco $(69,92 \mathrm{~g})$ y rendimiento comercial $(5,28 \mathrm{t} / \mathrm{ha})$; el tratamiento T1 también mejoró en las características físicas de calidad: peso de fruto fresco $(30,27 \mathrm{~g})$, peso de fruto $\operatorname{seco}(7,0 \mathrm{~g})$, longitud de fruto $(14,76 \mathrm{~cm})$, diámetro de fruto $(3,48 \mathrm{~cm})$.

Palabras claves: Dosis; Potasio; Ají Páprika, Capsicum annuum L.; Rendimiento.

\begin{abstract}
The objective was to determine what dose of potassium is adequate to obtain a higher yield of paprika pepper in the district and province of Barranca. Four blocks and four treatments were installed: $\mathrm{T} 1=200, \mathrm{~T} 2=150, \mathrm{~T} 3=100$ and T4 $=0 \mathrm{~kg} / \mathrm{ha}$. The Completely Random Block Design was used and the analysis of variance was performed at $5 \%$. Treatment $\mathrm{T} 1$ excelled in the physical characteristics of the paprika pepper: plant height $(77,95 \mathrm{~cm})$, number of fruits per plant (15 units), weight of the dried fruit $(69,92 \mathrm{~g})$ and commercial yield $(5.28 \mathrm{t} / \mathrm{ha})$; Treatment T1 also improved in the physical quality characteristics: weight of fresh fruit $(30,27 \mathrm{~g})$, weight of dried fruit $(7.0 \mathrm{~g})$, length of fruit $(14,76 \mathrm{~cm})$, diameter of fruit $(3,48 \mathrm{~cm})$.
\end{abstract}

Keywords: Dose; Potassium; Ají Paprika, Capsicum annuum L.; Performance.

\section{INTRODUCCIÓN}

Durante muchos años el agricultor ha empleado de manera inadecuada el fertilizante a base de potasio, esto ha ocasionado menor rendimiento, elevado costo, contaminación en el suelo y por ende pérdida económica. Esta afirmación se sostiene con Alconada et al. (2015) quienes manifiestan que a niveles excesivos de potasio pueden causar antagonismos que llevan a deficiencias de otros nutrientes como el magnesio o el calcio. En este sentido, la Organización de las Naciones Unidas para la Alimentación y la Agricultura FAO (2002) establece que una deficiencia de potasio ocasiona que los frutos sean pequeños, pueden tener lesiones o puntos dañados, hay un pobre almacenamiento y mantenimiento de la calidad.

Debido a esta variación de fertilización de potasio por los agricultores de la provincia de Barranca se ha realizado investigaciones relacionadas a la dosis de potasio, según Romero et. al. (2016), expone que el manejo adecuado de la nutrición de un cultivo implica hacer un uso eficiente de los nutrientes. Por tanto, esta investigación tuvo como objetivo determinar la eficiencia agronómica y de recuperación del fertilizante para nitrógeno, fósforo y potasio en ají habanero y ají tabasco. Los tratamientos fueron T1: Testigo, T2: fertilización según el programa que maneja el Centro Experimental de la Universidad Nacional de Colombia (CEUNP) (N: 150, P2O5:100 y K2O:300), T3: programa de fertilización de CEUNP + $50 \%$ y el T4: programa de fertilización de CEUNP + 100\%. Los mejores rendimientos de tabasco y habanero se presentan en el T4 y los menores en el T1.

Por otro lado, Martínez (2015), realizó ensayo manejando variaciones de Nitrógeno, Fósforo, Potasio, Calcio y Magnesio, tomando como testigo la solución de Hoagland y Arnon, con el objetivo de seleccionar los requerimientos nutricionales del cultivo de Capsicum Annuum L. variedad Cayenne. Las concentraciones de elementos nutritivos en cada etapa presentaron variaciones significativas. Se seleccionaron dos mejores tratamientos $\mathrm{T} 1(\mathrm{~N} 2 \mathrm{O}=2, \mathrm{P} 2 \mathrm{O} 5=3, \mathrm{~K} 2 \mathrm{O}=46, \mathrm{Ca}=2 \mathrm{y}$ $\mathrm{Mg}=0), \mathrm{T} 2=(\mathrm{N} 2 \mathrm{O}=3, \mathrm{P} 2 \mathrm{O} 5=5, \mathrm{~K} 2 \mathrm{O}=56, \mathrm{Ca}=3 \mathrm{y} \mathrm{Mg}=0), \mathrm{T} 3$ $(\mathrm{N} 2 \mathrm{O}=12, \mathrm{P} 2 \mathrm{O} 5=9, \mathrm{~K} 2 \mathrm{O}=24, \mathrm{Ca}=36$ y Mg $=2.4)$ y testigo $\mathrm{T} 4$ $(\mathrm{N} 2 \mathrm{O}=0, \mathrm{P} 2 \mathrm{O} 5=0, \mathrm{~K} 2 \mathrm{O}=0, \mathrm{Ca}=0$ y Mg = 0). De acuerdo a los resultados de número de frutos por planta, peso de frutos $y$ rendimiento por hectárea, se seleccionó el T1 como el mejor para ser manejado como plan de fertilización, reduciendo de forma significativa el uso de fertilizantes y los costos de producción

FAO, (1992), expone que el Potasio (K), que suple de 1 a 4

Recibido:08/10/2020 - Aprobado:17/11/2020

${ }^{1}$ Director Colegio 21571 Ricardo Palma Soriano-Hacienda Araya Grande. Barranca, Perú.

${ }^{2}$ Universidad Nacional José Faustino Sanchez Carrion - Huacho (Lima-Perú)

${ }^{3}$ Universidad Autónoma del Perú-Lima (Lima Perú) 
porcentualmente del extracto seco de la planta, tiene muchas funciones. Activa más de 60 enzimas (substancias químicas que regulan la vida). Por ello juega un papel vital en la síntesis de carbohidratos y de proteínas. El K mejora el régimen hídrico de la planta y aumenta su tolerancia a la sequía, heladas y salinidad. Las plantas bien provistas con $\mathrm{K}$ sufren menos de enfermedades.

Se planteó los siguientes objetivos estudiar el efecto de los tratamientos en la producción de frutos de la variedad de ají escabeche El trasplante, se realizó con plantas con 5 hojas funcionales. Los tratamientos investigados fueron $T_{1} \cdot 280-180-$ $300 \mathrm{~T}_{2}$. 240-160-260; $\mathrm{T}_{3}$. 200-140-220 y T. 160-120-180 de urea, Di fosfato de amonio y sulfato de potasio respectivamente y $T_{5}$ testigo absoluto. El ají escabeche de tipo arbustivo, respondió a la fertilización potenciando el crecimiento en altura, para alcanzar la mayor altura con el nivel de fertilización 240-160-260. En el número de frutos por planta, la mayor productividad, le correspondió al tratamiento $\mathrm{T}_{2}$ con 240-160-260. El tratamiento que alcanzó los mejores rendimientos (14,32 t/ha.) correspondió al tratamiento $T_{2}$ (Vélez, 2015).

Debido a esta situación sobre la fertilización inadecuada de potasio en el cultivo de ají paprika variedad Papri King se realizó la investigación con el objetivo de determinar que dosis de potasio es adecuado para el rendimiento y calidad en el distrito y provincia de Barranca

\section{MATERIALES Y MÉTODOS}

\section{Análisis de varianza}

Obtenidos los datos en campo y laboratorio de desarrolló el análisis estadístico de análisis de varianza y el resultado se comparó con los valores de la distribución de Fisher al $5 \%$ de error. De esta manera se definió si es significativo o no; es decir si hubo efecto de dosis de potasio. Mencionado este procedimiento se fundamenta con Fernández de la Fuente, (2011) quien expone que el análisis de varianza, el factor cuya influencia se quiere corroborar se introduce de forma discreta, independiente que sea de naturaleza continua o no. los componentes del análisis de varianza se indica en la tabla 1

Tabla 1. Componentes del análisis de varianza

\begin{tabular}{|c|c|c|c|c|c|c|}
\hline $\begin{array}{l}\text { Firtede } \\
\text { Vaizä́n }\end{array}$ & $S$ & 0 & OM & MotbolECM & $\begin{array}{l}\text { Natboll } \\
\text { ECM) }\end{array}$ & F.d \\
\hline Bopies & $\mathbb{S}_{b}$ & $b-1$ & $O M_{b}=O C b-1$ & $\frac{\sigma_{e+}^{2} \sum \beta_{i}^{2}}{(b-1)}$ & $\sigma_{e+}^{2} t \sigma_{\beta}^{2}$ & $\mathrm{CM}_{\mathrm{b}} / \mathrm{OM}_{\mathrm{e}}$ \\
\hline Trdaniertos & $\mathfrak{S}_{t}$ & $T-1$ & $\mathrm{OM}_{\mathrm{t}}=\mathrm{OC}+\mathrm{t}-1$ & $\frac{\sigma_{e}^{2}+b \sum T_{i}^{2}}{(t-1)}$ & $\sigma_{e+b}^{2} \sigma_{t}^{2}$ & $\mathrm{CM}_{\mathrm{t}} / \mathrm{OM}_{\mathrm{e}}$ \\
\hline $\begin{array}{l}\text { Eror } \\
\text { Tdad }\end{array}$ & $\begin{array}{l}S_{e} \\
S_{t}\end{array}$ & $\begin{array}{c}(b-1)(t-1) \\
t d-1\end{array}$ & $\left.C M_{e}=-\mathfrak{C},(b-1) t-1\right)$ & $\sigma_{\theta}^{2}$ & $\sigma_{e}^{2}$ & \\
\hline
\end{tabular}

Fuente: Núñez et. al. (2007) “Regresión y Análisis de Varianza” España

\section{Prueba de Duncan}

Luego de operar con el análisis de varianza se aplicó la prueba de hipótesis de Duncan a los parámetros que resultaron significativos. Pues con esta prueba de hipótesis determinó que tratamiento sobresale y su diferenciación. Dicho esta afirmación se sostiene con Fallas (2012), quien manifiesta que la prueba de Duncan ajusta la diferencia crítica considerando si los dos promedios que se comparan son adyacentes o sí por el contrario existe uno o más medias entre las medias que se están comparando.

\section{Tratamientos}

La investigación trata acerca de la aplicación de potasio, lo cual se empleó como Sulfato de Potasio $\left(\mathrm{N}=0, \mathrm{P}_{2} \mathrm{O}_{5}=0\right.$ y $\left.\mathrm{K}_{2} \mathrm{O}=50\right)$ y se tuvo en cuenta la dosis usada por los agricultores del distrito de Barranca y el testigo. Cabe mencionar las fuentes de nitrógeno Urea $\left(\mathrm{N}=46, \mathrm{P}_{2} \mathrm{O}_{5}=0\right.$ y $\left.\mathrm{K}_{2} \mathrm{O}=0\right)$ y Fosfato Diamónico $\left(\mathrm{N}=18, \mathrm{P}_{2} \mathrm{O}_{5}=46\right.$ y $\left.\mathrm{K}_{2} \mathrm{O}=00\right) \mathrm{A}$ continuación en la tabla 2 se detalla los tratamientos.

Tabla 2. Aplicación de dosis de potasio en el cultivo de ají páprika..

\begin{tabular}{ll}
\hline TRATAMIENTO & Dosis de potasio Kg./ ha \\
\hline$T_{1}$ & $200-100-200$ \\
$T_{2}$ & $200-100-150$ \\
$T_{3}$ & $200-100-100$ \\
$T_{4}$ & $200-100-0.0$
\end{tabular}

\section{RESULTADOS}

\section{Altura de planta}

De acuerdo a los resultados mediante el análisis de varianza se determinó que el $T_{1}$ sobresalió con $77,95 \mathrm{~cm}$ con relación a los demás; sin embargo, no son significativos lo cual indica que no hubo efecto de dosis de potasio siendo todos los tratamientos estadísticamente homogéneos (Ver tabla 4 y figura 1)

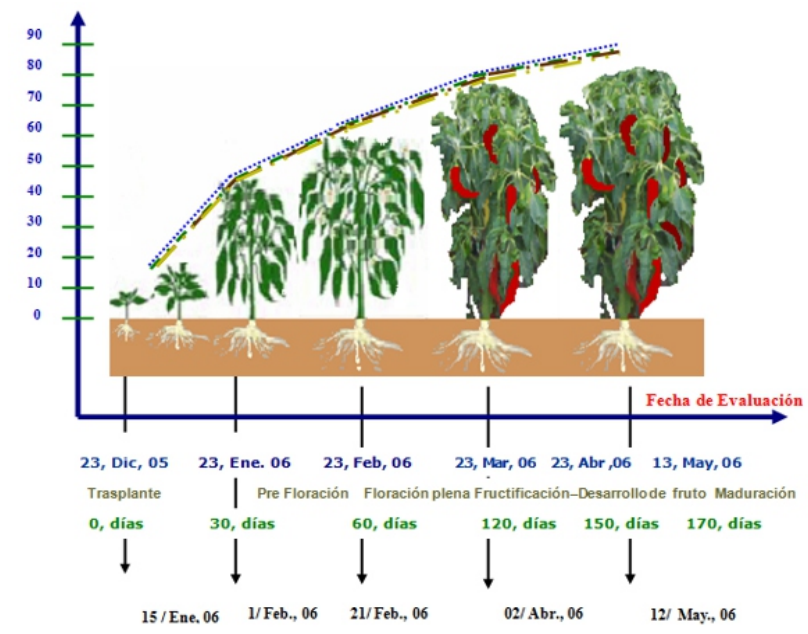

Figura 1. Altura de planta de acuerdo a las dosis de potasio

Tabla 3. Promedios de altura de planta (cm.), según las fechas de evaluaciones.

\begin{tabular}{ccccc}
\cline { 2 - 5 } & \multicolumn{5}{c}{ TRATAMIENTO } \\
\cline { 2 - 5 } Fecha de evaluación & \multicolumn{1}{c}{$\mathrm{T}_{1}$} & \multicolumn{1}{c}{$\mathrm{T}_{2}$} & \multicolumn{1}{c}{$\mathrm{T}_{3}$} & $\mathrm{~T}_{4}$ \\
& $\square$ & $\square$ & $\square$ & $\square$ \\
\hline $15-01-06$ & 16,70 & 16,40 & 16,23 & 16,15 \\
$01-02-06$ & 45,62 & 45,47 & 45,15 & 45,05 \\
$21-02-06$ & 56,23 & 55,48 & 55,15 & 55,05 \\
$02-04-06$ & 73,48 & 72,23 & 69,68 & 67,95 \\
$12-05-06$ & 77,95 & 76,90 & 75,08 &, 33 \\
\hline
\end{tabular}




\section{Número de frutos por planta}

Respecto a la cantidad de frutos por planta el $T_{1}$ obtuvo 15 frutos, lo cual se diferencia con los demás. No obstante, no hubo significancia; es decir no influyó el potasio siendo estadísticamente homogéneos. Asimismo, el coeficiente de variación es de $13.45 \%$, esto se interpretación que hay una ligera variación entre promedios de parcela. (Ver tabla 4)

\section{Peso seco de frutos por planta}

En el peso de frutos secos que se indica los resultados en la tabla 3 , se determinó por medio del análisis de varianza que no hay diferencias significativas en los tratamientos. Por lo tanto, este resultado se interpreta que no hubo efecto de dosis de potasio (Ver tabla 4).

\section{Rendimiento comercial}

Respecto a los resultados de rendimiento comercial se determinó por medio de los análisis estadísticos de análisis de varianza que no hay significancia estadística, lo cual quiere decir que no influyó la dosis de potasio. También se indica que el coeficiente de variación es de $17.75 \%$, lo que quiere decir que hay variación de los promedios (Ver tabla 4).

Tabla 4. Características físicas de ají paprika variedad papri King

\begin{tabular}{|c|c|c|c|c|c|c|}
\hline Tratamiento & $\begin{array}{c}\text { Dosis de } \mathrm{K} \\
\mathrm{Kg} . / \mathrm{Ha} \text {. }\end{array}$ & $\begin{array}{l}\text { Altura de } \\
\text { planta } \\
\text { (cm.) }\end{array}$ & $\begin{array}{l}\text { Número de } \\
\text { frutos } \\
\text { (N/Planta) }\end{array}$ & $\begin{array}{l}\text { Peso fresco } \\
\text { de frutos } \\
\text { (No/Planta) }\end{array}$ & $\begin{array}{l}\text { peso seco } \\
\text { de frutos } \\
\text { (N/Planta) }\end{array}$ & $\begin{array}{l}\text { Rendimiento } \\
\text { comercial } \\
\text { (Tm/ha). }\end{array}$ \\
\hline $\mathrm{T} 1$ & 200 & 77,95 a & $12,25 \mathrm{a}$ & 293,68 a & 69,920 a & 5,282 a \\
\hline $\mathrm{T} 2$ & 150 & 76,90 a & $13,75 a$ & 276,70 a & 65,878 a & $4,976 \mathrm{a}$ \\
\hline T3 & 100 & 75,08 a & 13,00 a & 294,48 a & 60,478 a & 4,569 a \\
\hline T4 & 0.0 & $73,33 \mathrm{a}$ & $12,50 \mathrm{a}$ & 249,48 a & 59,398 a & $4,487 \mathrm{a}$ \\
\hline Significacion & & ** & $* *$ & ** & ** & ** \\
\hline \multicolumn{2}{|c|}{ Coeficiente de variacion(\%) } & 8,09 & 13,45 & 17,74 & 8,10 & 17,75 \\
\hline
\end{tabular}

\section{Peso de un fruto seco}

Respecto a la evaluación de un peso de fruto seco, los resultados del análisis de varianza determinaron que no hubo efecto de dosis de potasio; siendo no significativo en los tratamientos. Cabe resaltar que el coeficiente de variación es de $6,62 \%$ que significa una ligera variación de promedios de parcelas (Ver tabla 4)

\section{Longitud de fruto}

Concerniente al tamaño de ají paprika, por medio de las operaciones de análisis de varianza determinaron que no hubo variación estadística; por lo tanto, no influyeron las dosis de potasio en los tratamientos. Se estimó también el coeficiente de variación de $4.33 \%$ que lo cual quiere decir que la variación de promedios de parcela es leve (ver tabla 4).

Tabla 5. Características de calidad de ají páprika var. Papri King

\begin{tabular}{|c|c|c|c|c|c|c|}
\hline Tratamiento & $\begin{array}{c}\text { Dosis de K } \\
\mathrm{Kg} . / \mathrm{Ha} .\end{array}$ & $\begin{array}{l}\text { Peso un } \\
\text { frutos fresco } \\
\text { (g) }\end{array}$ & $\begin{array}{l}\text { Peso de un } \\
\text { frutos seco } \\
\text { (g) }\end{array}$ & $\begin{array}{l}\text { longitud de } \\
\text { fruto } \\
(\mathrm{cm})\end{array}$ & $\begin{array}{l}\text { Diámetro de } \\
\text { fruto } \\
(\mathrm{cm})\end{array}$ & $\begin{array}{c}\text { Grados Asta } \\
\text { (u). }\end{array}$ \\
\hline T1 & 200 & 30,27 a & 7,00 a & 14,76 a & 3,48 a & $270,00 \mathrm{a}$ \\
\hline T2 & 150 & $28,88 \mathrm{a}$ & 6,86 a & $14,72 \mathrm{a}$ & 3,39 a & 263,25 a \\
\hline T3 & 100 & $28,22 \mathrm{a}$ & $6,61 \mathrm{a}$ & $14,58 \mathrm{a}$ & 3,36 a & $258,00 \mathrm{a}$ \\
\hline T4 & 0.0 & 27,86 a & 6,59 a & $14,52 \mathrm{a}$ & 3,33 a & $250,00 \mathrm{a}$ \\
\hline Significacion & & ** & ** & ** & ** & ** \\
\hline \multicolumn{2}{|c|}{ Coeficiente de variacion(\%) } & 5,90 & 7,62 & 4,33 & 2,58 & 5,31 \\
\hline
\end{tabular}

\section{DISCUSIÓN}

De acuerdo a los resultados de la tabla 4, indica que la mayor altura de planta obtuvo el $T_{1}$ con $77,95 \mathrm{~cm}$ con relación a los demás, también se determinó mediante análisis de varianza que no son significativos; es decir que no influyó las dosis de potasio. Por lo tanto, la fórmula $\mathrm{N}_{2}=200 ; \mathrm{P}_{2} \mathrm{O}_{5}=100$ y $\mathrm{K}_{2} \mathrm{O}=200$ sobresalió en mayor presencia porte y altura de ají paprika. Interpretado estos resultados se sostiene con Vélez (2015), quien estudió el efecto de los tratamientos en la producción de frutos de la variedad de ají escabeche. Los tratamientos fueron $\mathrm{T}_{1}$.280-180-300; $\mathrm{T}_{2}$. 240-160-260; $\mathrm{T}_{3}$. 200-140-220 y $\mathrm{T}_{4} .160-$ 120-180 y $\mathrm{T}_{5}$ testigo absoluto. Respondió la fertilización con
NPK potenciando el crecimiento en altura con el nivel de fertilización $\mathrm{T}_{2}$ (240-160-260).

Concerniente al número de frutos por planta, se precisó que el $\mathrm{T}_{1}$ obtuvo 15 frutos lo cual se diferencia a los demás tratamientos. De la misma manera se precisa que no hubo diferencias significativas; es decir no influyeron las dosis de potasio (Ver tabla 4). Por lo tanto, a esta dosis de $\mathrm{N}_{2}=200 ; \mathrm{P}_{2} \mathrm{O}_{5}=$ 100 y $\mathrm{K}_{2} \mathrm{O}=200$, obtuvo mayor rendimiento. Analizado este resultado se contrasta con el desarrollado por Martínez (2015), quien realizó un ensayo con el objetivo de seleccionar los requerimientos nutricionales de Capsicum Annuum L. variedad Cayenne. Los tratamientos $\mathrm{T}_{1=}\left(\mathrm{N}_{2} \mathrm{O}=2, \mathrm{P}_{2} \mathrm{O}_{5}=3, \mathrm{~K}_{2} \mathrm{O}=46, \mathrm{Ca}=2\right.$ 
y $\mathrm{Mg}=0), \mathrm{T}_{2}=\left(\mathrm{N}_{2} \mathrm{O}=3, \mathrm{P}_{2} \mathrm{O}_{5}=5, \mathrm{~K}_{2} \mathrm{O}=56, \mathrm{Ca}=3\right.$ y $\left.\mathrm{Mg}=0\right), \mathrm{T}_{3}$ $=\left(\mathrm{N}_{2} \mathrm{O}=12, \mathrm{P}_{2} \mathrm{O}_{5}=9, \mathrm{~K}_{2} \mathrm{O}=24, \mathrm{Ca}=36 \mathrm{y} \mathrm{Mg}=2.4\right) \mathrm{y}$ testigo $\mathrm{T}_{4}=$ $\left(\mathrm{N}_{2} \mathrm{O}=0, \mathrm{P}_{2} \mathrm{O}_{5}=0, \mathrm{~K}_{2} \mathrm{O}=0, \mathrm{Ca}=0\right.$ y $\left.\mathrm{Mg}=0\right)$. De acuerdo a los resultados de número de frutos por planta, peso de frutos y rendimiento por hectárea, se seleccionó el $\mathrm{T}_{1}$ como el mejor para el plan de fertilización.

En cuanto al peso seco de frutos por planta en la tabla 4, se indica que el $\mathrm{T}_{1}$ obtuvo mayor peso con 69,92 g por planta. También se determinó que no hay diferencias significativas en los tratamientos; por lo tanto, no hubo efecto de dosis de potasio. Este resultado quiere decir que a esta dosis de $\mathrm{N}_{2}$ $=200 ; \mathrm{P}_{2} \mathrm{O}_{5}=100$ y $\mathrm{K}_{2} \mathrm{O}=200$ favoreció en mayor peso de frutos seco; ya que el potasio influyó en la formación de carbohidratos, estrés ambiental y enfermedades. Analizado este resultado se sustenta con FAO (1992), expone que el Potasio suple de 1 al $4 \%$ del extracto seco de la planta, tiene muchas funciones. Activa más de 60 enzimas (substancias químicas que regulan la vida). Por ello juega un papel vital en la síntesis de carbohidratos y de proteínas. Las plantas bien provistas con potasio sufren menos de enfermedades.

Respecto al de rendimiento comercial que se detalla en la tabla 4 , se indica que el $\mathrm{T}_{1}$ con dosis de $\mathrm{N}_{2}=200, \mathrm{P}_{2} \mathrm{O}_{5}=100$ y $\mathrm{K}_{2} \mathrm{O}=$ 200 sobresalió con $5,282 \mathrm{tn} /$ ha, este resultado quiere decir que a esta dosis de potasio es favorable; por lo que incrementó $15,05 \%$ con relación al testigo. Analizado este resultado se sostiene con Romero et. al. (2016), expone que su investigación tuvo como objetivo determinar la eficiencia agronómica y de recuperación del fertilizante para nitrógeno, fósforo y potasio en ají habanero y ají tabasco. Los tratamientos fueron $\mathrm{T}_{1}$ : Testigo, $\mathrm{T}_{2}$ : Programa que maneja el Centro Experimental de la Universidad Nacional de Colombia (CEUNP) (N: 150, $\mathrm{P}_{2} \mathrm{O}_{5}: 100$ y $\left.\mathrm{K}_{2} \mathrm{O}: 300\right), \mathrm{T}_{3}$ : programa de fertilización de CEUNP $+50 \%$ y el $\mathrm{T}_{4}$ : programa de fertilización de CEUNP + $100 \%$. Los mejores rendimientos de tabasco y habanero se presentan en el $\mathrm{T}_{4} \mathrm{y}$ los menores en el $\mathrm{T}_{1}$.

En cuanto a la evaluación de laboratorio, se hicieron las mediciones de longitud de ají paprika determinándose que el $\mathrm{T}_{1}$ con dosis de $\mathrm{N}_{2}=200, \mathrm{P}_{2} \mathrm{O}_{5}=100$ y $\mathrm{K}_{2} \mathrm{O}=200$ sobresalió con $14,76 \mathrm{~cm}$, también es necesario mencionar que no hubo diferencias estadísticas; es decir hay homogeneidad entre los tratamientos (Ver tabla 5). Debido a este resultado se define qua el $T_{1}$ resaltó con esta fórmula en tamaño y presencia de ají paprika, lo cual favorece a su comercialización. Este análisis es corroborarle con Vélez (2015), quien estudió el efecto de los tratamientos en la producción de ají escabeche. Los tratamientos fueron $T_{1} .280-180-300 ; T_{2} .240-160-260 ; T_{3}$. 200140-220 y $\mathrm{T}_{4} \cdot 160-120-180$ y $\mathrm{T}_{5}$ testigo absoluto. Determinándose que el $\mathrm{T}_{1}$ resaltó con $12.9 \mathrm{~cm}$ en relación a los demás.

Respecto al peso de un fruto seco, los resultados de la tabla 5 determinaron que el $\mathrm{T}_{1}$ obtuvo $7 \mathrm{~g}$., lo cual se demuestra que esta fórmula de $\mathrm{N}_{2}=200, \mathrm{P}_{2} \mathrm{O}_{5}=100$ y $\mathrm{K}_{2} \mathrm{O}=200$ resaltó en rendimiento; siendo está dosis favorable para la formación de carbohidratos, presencia de fruto y elasticidad. Analizado esta investigación se basa con FAO (1992), expone que el Potasio suple de 1 a $4 \%$ del extracto seco de la planta, tiene muchas funciones. Activa más de 60 enzimas (substancias químicas que regulan la vida). Por ello juega un papel vital en la síntesis de carbohidratos y de proteínas.

\section{CONCLUSIONES}

Se determinó que la dosis de $\mathrm{T}_{1}$ con la fórmula de $\mathrm{N}_{2} \mathrm{O}=200$, $\mathrm{P}_{2} \mathrm{O}_{5}=100$ y $\mathrm{K}_{2} \mathrm{O}=200 \mathrm{~kg} / \mathrm{ha}$ obtuvo mayor rendimiento comercial con $5.282 \mathrm{tn} / \mathrm{ha}$ y el testigo $\left(\mathrm{N}_{2} \mathrm{O}=0, \mathrm{P}_{2} \mathrm{O}_{5}=100 \mathrm{y}\right.$ $\mathrm{K}_{2} \mathrm{O}=200 \mathrm{~kg} / \mathrm{ha}$ ) con $4.487 \mathrm{tn} / \mathrm{ha}$ diferenciándose con $0.795 \mathrm{tn} /$ ha.

Respecto a las evaluaciones de altura de planta, número de frutos por planta, peso seco de frutos por planta, rendimiento comercial no hubo significancia estadística; es decir no influyeron las dosis de potasio en los tratamientos.

También se determinó que a esta dosis de potasio con $\mathrm{N}_{2} \mathrm{O}=$ $200, \mathrm{P}_{2} \mathrm{O}_{5}=100$ y $\mathrm{K}_{2} \mathrm{O}=200 \mathrm{~kg} / \mathrm{ha}$, influyó favorablemente en las variables de calidad como peso de un fruto seco y longitud de fruto obteniéndose presencia que favorece a la comercialización.

\section{RECOMENDACIONES}

Es importante realizar investigaciones utilizando esta dosis de potasio en otras variedades de ají páprika como sonora, Lorca, Jaranda, Papriqueen con el motivo de determinar la fertilización adecuada para tener mayor rendimiento y calidad. Es necesario tomar muestras de suelo de manera escalonada antes de la siembra con el fin de determinar mediante análisis de suelo que dosis es adecuado, para la fertilización de ají páprika y de esta manera evitar el estrés de la planta, el exceso de costo y reducir la contaminación en el suelo.

Cabe resaltar que a esta dosis de $\mathrm{N}_{2} \mathrm{O}=200, \mathrm{P}_{2} \mathrm{O}_{5}=100$ y K${ }_{2} \mathrm{O}=$ $200 \mathrm{~kg} / \mathrm{ha}$, favoreció en el rendimiento y calidad de ají paprika; por lo tanto, se recomienda aplicar esta fórmula en el distrito de Barranca. 


\section{REFERENCIAS.}

Alconada M., Lanfranco, J., \& Pellegrini, A. (2015). Suelo en el paisaje. Edulp, 1,1-154.

Fallas, J. (2012), "Análisis De Varianza Comparando Tres o Más Medias", Manual estadístico. Universidad Nacional de Costa Rica. Costa Rica. Pag. 17.

FAO (1992), "Los fertilizantes y su uso" FAO (Organización de las Naciones Unidas para la Alimentación y la Agricultura), IFA (Asociación Internacional de los Fertilizantes). Manual Mundial sobre el Uso de Fertilizantes, Roma- Italia. Pag. 8 y 54

Fernández de la Fuente, S. (2011), "Análisis de varianza. Libro. Gestión Aeronáutica, Facultad de Ciencias Económicas y Empresariales. Universidad Autónoma de Madrid, España. Pag. 2.

Martínez, A., (2015), "Requerimientos nutricionales del ají Capsicum annuum L. y su relación con rendimiento bajo condiciones ambientales de Palmira, Valle del Cauca", Tesis o trabajo de investigación presentado como requisito parcial para optar al título de: Magister en Ciencias Agrarias, Línea de Investigación en Suelos. Universidad Nacional de Colombia.
Organización de las Naciones Unidas para la Alimentación y la Agricultura (FAO). (2002). Los fertilizantes y su uso.

Romero, M.; Encino, C.; García, S.; Wagner J.; Puentes, Y. y Menjivar, J. (2016), "Eficiencia de uso de nutrientes en ají tabasco (Capsicum frutescens L.) y habanero (Capsicum chínense Jacq). Artículo científico. Revista de Investigación y Agraria volumen 7 Número 2. Ambiental. Universidad Nacional de Colombia, Sede Palmira. Colombia. Pag. 121.

Vélez, L., (2015), "Respuesta del ají escabeche (Capsicum baccatum L.) a cuatro dosis Diferentes de Fertilizantes en la Granja Experimental Santa Inés". Trabajo de titulación sometida a consideración del $\mathrm{H}$. Consejo Directivo de la Unidad Académica de Ciencias Agropecuarias como Requisitos Previo para Optar al Grado de: Ingeniero Agrónomo. Universidad Técnica de Machala. Ecuador. Pag. 18. 